Advances in Dynamical Systems and Applications (ADSA).

ISSN 0973-5321, Volume 14, Number 2, (2019). 245.255

(C) Research India Publications

https://dx.doi.org/10.37622/ADSA/14.2.2019.245-255

\title{
Dynamics of Two Dimensional Piecewise Smooth Maps with Stochastically Varying Border
}

\author{
Dhrubajyoti Mandal* \\ Department of Mathematics, \\ BITS Pilani K.K. Birla Goa Campus, \\ Zuarinagar, Goa-403726, India
}

\begin{abstract}
Basin of attraction of an attractor plays a significant role in determining the dynamics of any system. We are commonly familiar with the deterministic nature of the basin of attraction of an attractor. However, under some special conditions this deterministic nature may change and as a result of that nondeterministic or probabilistic basins of attraction may be observed. This paper shows the occurrence of probabilistic basins of attraction in nonsmooth maps. We have shown that if the border of a class of nonsmooth maps vibrates stochastically inside a small bounded region of the phase space, retaining deterministic dynamics on each compartment of the phase space, then there may exist probabilistic basins of attraction in such systems. In that case, a part of the basin of attraction of the existing attractor may be converted into a non-deterministic basin as the attractor approaches towards the region of variation of the border, upon the variation of some system parameters. Therefore, such a phenomenon can occur in engineering or physical systems which are modeled by nonsmooth maps, which may ultimately lead to the loss of stability of such systems.
\end{abstract}

Keyword: Two dimensional piecewise smooth maps; Border collision normal form; Basin of attraction; Stochastically vibrating border.

*corresponding author 


\section{INTRODUCTION}

Piecewise smooth (PWS) maps are used frequently in modelling many physical and engineering systems [1, 2, 3, 4, 6, 9, 5, 10]. The dynamics of these physical systems may be contaminated by noise as these systems always exist in noisy environments, and therefore the dynamics of such systems should be studied considering the effect of various possible noise terms that can have a significant effect on the system dynamics. Most of such studies existing in the literature assume that only the subsequent iterates of the PWS maps are affected by the noise and the position of the border remains unaltered.

However there may exist many kinds of noise which may affect the system dynamics in such a way that the position of the border do not remain fixed, rather it varies stochastically inside a small region, retaining the deterministic dynamics on each compartment of the phase space [7]. This type of varying borders may occur due to some mechanical origin (loosely fitted panels or dividers), as a result of environmental fluctuations (effects of change of pressure or temperature), due to some defined rule of measurement (e.g., if in the measurement technique a transition takes place if the observed data crosses some tolerance band). In all these cases we have to consider the stochastic movement of the switching surface, in a bounded region of the phase space. Glendinning showed that the attractor of a two dimensional PWS map is the same as the attractor of an iterated function system subject to the condition that the considered map is a contraction mapping in absence of noise. Some other possibilities of the dynamics has also been investigated numerically in the absence of such assumptions [7]. Besides this, the effect of stochastically moving border on basins of attraction in a class of piecewise smooth one dimensional map has also been studied. It has been shown that a part of the deterministic basin of attraction of the considered class of system gets converted into a probabilistic basin of attraction (any two orbits starting from the same initial point may have different dynamics, i.e., one of them may converge to the stable fixed point whereas the other may diverge to infinity) due to the presence of such stochastically moving border [8].

In this paper, we have considered a class of two dimensional PWS continuous map with stochastically varying switching manifold, maintaining deterministic dynamics in the two compartments separated by the switching surface. The PWS map has been considered to have a stable fixed point on any one compartment of the phase space. Now if we consider random variation of the switching manifold in a small bounded region about its deterministic location (this region does not contain the stable fixed point), then the border collision normal form becomes a PWS discontinuous map. Now if the basin of attraction of the stable fixed point intersects this region of variation of 
the border, in that case this region of intersection may act as a non-deterministic basin of attraction. In this case, both convergence to the stable fixed point and convergence to some other attractor (including attractor at infinity) of orbits, starting from the same initial point lying inside this probabilistic basin, will have non-zero probabilities.

\section{MATHEMATICAL FORMULATION}

We consider the border collision normal form of a class of two dimensional piecewise smooth map given as follows.

$$
\left(\begin{array}{l}
x_{n+1} \\
y_{n+1}
\end{array}\right)= \begin{cases}\left(\begin{array}{cc}
\tau_{L} & 1 \\
-\delta_{L} & 0
\end{array}\right)\left(\begin{array}{l}
x_{n} \\
y_{n}
\end{array}\right)+\left(\begin{array}{l}
1 \\
0
\end{array}\right) \mu, & \text { if } x_{n} \leq 0, \\
\left(\begin{array}{cc}
\tau_{R} & 1 \\
-\delta_{R} & 0
\end{array}\right)\left(\begin{array}{l}
x_{n} \\
y_{n}
\end{array}\right)+\left(\begin{array}{l}
1 \\
0
\end{array}\right) \mu, & \text { if } x_{n} \geq 0,\end{cases}
$$

where $\tau_{L}, \tau_{R}$ and $\delta_{L}, \delta_{R}$ are constants. Let us denote the compartments $\left\{(x, y) \in \mathbb{R}^{2}\right.$ : $x \leq 0\}$ by $L$ and $\left\{(x, y) \in \mathbb{R}^{2}: x \geq 0\right\}$ by $R$. The fixed points of the system are given by

$$
\mathbf{L}^{*}=\left(L_{x}^{*}, L_{y}^{*}\right)=\left(\frac{\mu}{1-\tau_{L}+\delta_{L}}, \frac{-\delta_{L} \mu}{1-\tau_{L}+\delta_{L}}\right)
$$

and

$$
\mathbf{R}^{*}=\left(R_{x}^{*}, R_{y}^{*}\right)=\left(\frac{\mu}{1-\tau_{R}+\delta_{R}}, \frac{-\delta_{R} \mu}{1-\tau_{R}+\delta_{R}}\right)
$$

respectively. These two fixed point exist if $L_{x}^{*} \leq 0$ and $R_{x}^{*}>0$. On the other hand if $L_{x}^{*} \geq 0$, then $\mathbf{L}^{*}$ becomes a virtual fixed point of (1). Similarly if $R_{x}^{*} \leq 0$, then $\mathbf{R}^{*}$ is a virtual fixed point of the map (1).

Denote the matrices

$$
\left(\begin{array}{cc}
\tau_{L} & 1 \\
-\delta_{L} & 0
\end{array}\right) \text { and }\left(\begin{array}{cc}
\tau_{R} & 1 \\
-\delta_{R} & 0
\end{array}\right)
$$

by $\mathbf{A}_{L}$ and $\mathbf{A}_{R}$ respectively. Let the eigenvalues of $\mathbf{A}_{L}$ be $\lambda_{1 L}, \lambda_{2 L}$ and the eigenvalues of $A_{R}$ be $\lambda_{1 R}, \lambda_{2 R}$. Again if the values of $\tau_{L}, \tau_{R}, \delta_{L}, \delta_{R}$ be such that $\left(1-\tau_{L}+\delta_{L}\right)>0$ then as long as $\mu<0, L^{*}$ remains a fixed point of (1). Moreover we assume that $\lambda_{1 L}$ and $\lambda_{2 L}$ are such that $\mathbf{L}^{*}$ is a stable fixed point ${ }^{1}$. Now if we consider that the switching manifold $x=0$ moves stochastically inside the region $R_{0}=[-\varepsilon, \varepsilon] \times \mathbb{R} \subset \mathbb{R}^{2}$, then

\footnotetext{
${ }^{1}$ Here without loss of generality we assume that $\mathbf{L}^{*}$ exists as a stable fixed point. However it is also possible that instead of $\mathbf{L}^{*}, \mathbf{R}^{*}$ exists as a stable fixed point.
} 
under the assumption that the switching manifold remains as a continuous graph over $y$, the border collision normal form of (1) becomes

$$
\left(\begin{array}{l}
x_{n+1} \\
y_{n+1}
\end{array}\right)= \begin{cases}\left(\begin{array}{cc}
\tau_{L} & 1 \\
-\delta_{L} & 0
\end{array}\right)\left(\begin{array}{l}
x_{n} \\
y_{n}
\end{array}\right)+\left(\begin{array}{l}
1 \\
0
\end{array}\right) \mu, & \text { if } x_{n}<g_{n}(y), \\
\left(\begin{array}{cc}
\tau_{R} & 1 \\
-\delta_{R} & 0
\end{array}\right)\left(\begin{array}{l}
x_{n} \\
y_{n}
\end{array}\right)+\left(\begin{array}{l}
1 \\
0
\end{array}\right) \mu, & \text { if } x_{n} \geq g_{n}(y),\end{cases}
$$

where there exist an $\varepsilon>0$, such that for all $n$ and $y$, we have $\left|g_{n}(y)\right|<\varepsilon$. Also, without loss of generality, we have included the switching surface in a specific compartment. Now as $\mu \rightarrow 0$ the attractors of the map (2) remains confined in smaller and smaller region of the phase space and therefore we further assume that the distribution of $g_{i}(y)$ becomes independent of $y$ to the lowest order. This assumption implies that given any sequence $y_{i}, i=0,1,2, \cdots$ the values $g_{i}\left(y_{i}\right), i=0,1,2, \cdots$ are independent and identically distributed (iid) random variables, no matter how $y_{i}$ are chosen. In that case the above map (2) can further be rewritten as

$$
\left[\begin{array}{l}
x_{n+1} \\
y_{n+1}
\end{array}\right]=\left\{\begin{array}{lll}
\mathbf{A}_{L} \mathbf{X}_{n}+\mu \mathbf{C} & : & x_{n}<\xi_{n} \\
\mathbf{A}_{R} \mathbf{X}_{n}+\mu \mathbf{C} & : & x_{n} \geq \xi_{n}
\end{array}\right.
$$

where $\mathbf{A}_{L}=\left[\begin{array}{cc}\tau_{L} & 1 \\ -\delta_{L} & 0\end{array}\right], \mathbf{A}_{R}=\left[\begin{array}{cc}\tau_{R} & 1 \\ -\delta_{R} & 0\end{array}\right], \mathbf{X}_{n}=\left[x_{n}, y_{n}\right]^{T}$ and $\mathbf{C}=[1,0]^{T}$ and $\xi_{n}$ are independent and identically distributed (iid) real random variables satisfying $\left|\xi_{n}\right|<\varepsilon$.

Assume that the eigenvalues of $A_{L}$ and $A_{R}$ are real and distinct, i.e., $\lambda_{1 L} \neq \lambda_{2 L}$ and $\lambda_{1 R} \neq \lambda_{2 R}$. Then we can diagonalize both $\mathbf{A}_{L}$ and $\mathbf{A}_{R}$ as

$$
\mathbf{A}_{L}=\mathbf{P}_{L}^{-1} \mathbf{D}_{L} \mathbf{P}_{L}
$$

and

$$
\mathbf{A}_{R}=\mathbf{P}_{R}^{-1} \mathbf{D}_{R} \mathbf{P}_{R}
$$

where $\mathbf{D}_{L}=\left[\begin{array}{cc}\lambda_{1 L} & 0 \\ 0 & \lambda_{2 L}\end{array}\right], \mathbf{D}_{R}=\left[\begin{array}{cc}\lambda_{1 R} & 0 \\ 0 & \lambda_{2 R}\end{array}\right]$ and $\mathbf{P}_{L}, \mathbf{P}_{R}$ are two non-singular matrices.

Let us call the basin of attraction of $\mathbf{L}^{*}$ as $\mathcal{B}\left(\mathbf{L}^{*}\right)$. Now as $\mu \rightarrow 0, \mathbf{L}^{*} \rightarrow(0,0)$. Therefore, it is natural that for some parameter value of $\mu$ very close to 0 , the basin of attraction of $L^{*}$ should intersect the region of variation of the border. We consider such a condition where the basin of attraction of $\mathbf{L}^{*}$, i.e., $\mathcal{B}\left(\mathbf{L}^{*}\right)$, intersects the region of 
variation of the border, yet the fixed point itself remains outside that region. We define the region of intersection $R_{I}$ by

$$
R_{I}=\mathcal{B}\left(L^{*}\right) \bigcap\{(-\varepsilon, 0) \times \mathbb{R}\}
$$

Let us denote the map defined for $x_{n}<\xi_{n}$ and $x_{n} \geq \xi_{n}$ by $M_{L}$ and $M_{R}$ in (3). Now we also assume that the width of the narrow band (the region $R_{0}$ ), inside which the border moves stochastically, is so small compared to the stretching of the right hand map $M_{R}$ such that if an orbit starts from an initial point $\mathbf{X}_{0} \in R_{I}$ then there exists a finite number $n_{X_{0}}$, such that if the right hand side map $M_{R}$ is applied for $n_{X_{0}}$ times on the subsequent iterates repeatedly then the $n_{X_{0}}$-th iterate $\mathbf{X}_{n_{X_{0}}}$ goes out of the basin of attraction of the stable fixed point $\mathbf{L}^{*}$ as well as outside the region of variation of the border. Moreover all the future iterates remain in that region forever. In other words, we assume that for every $X_{0} \in R_{I}$ there exist a finite positive integer $n_{X_{0}}$ such that $M_{R}^{i}\left(\mathbf{X}_{0}\right) \in \mathcal{B}\left(\mathbf{L}^{*}\right) \cup R_{0}$ for all $i=1,2, \cdots,\left(n_{X_{0}}-1\right)$ and $M_{R}^{i}\left(\mathbf{X}_{0}\right) \notin \mathcal{B}\left(\mathbf{L}^{*}\right) \bigcup R_{0}$ for all $i \geq n_{\mathbf{X}_{0}}$.

Then the question is, what can we say about any orbit starting inside the region $R_{I}$ ? We shall show in this paper that $R_{I}$ becomes a non-deterministic basin of attraction of (3) in the sense that any orbit starting from an initial point lying inside this region may converge to the stable fixed point $\mathbf{L}^{*}$ as well as converge to some other attractor including the attractor at infinity. Moreover both these possibilities of convergence and non-convergence (to the stable fixed point $L^{*}$ ) have non-zero probabilities.

\section{MINIMUM ITERATIONS REQUIRED FOR AN ORBIT TO ESCAPE FROM THE REGION $R_{I}$ UNDER REPEATED APPLICATION OF THE MAP $M_{L}$}

Suppose an orbit of (3) starts from a point $\mathbf{X}_{0} \in R_{I}$. We now find the minimum number of iterations needed to escape from the region $R_{I}$, under repeated application of only the map $M_{L}$ defined for the compartment $\mathrm{L}$ of (1). Then the $n$-th iterate is given by

$$
\mathbf{X}_{n}=\mathbf{A}_{L}^{n} \mathbf{X}_{0}+\mu \frac{\mathbf{I}-\mathbf{A}_{L}^{n}}{\mathbf{I}-\mathbf{A}_{L}} C
$$

Where $\mathbf{X}_{n}=\left[x_{n}, y_{n}\right]^{T}$. Now let $\mathbf{B}_{L}=\left(\mathbf{I}-\mathbf{A}_{L}\right)^{-1}$, then we have

$$
\mathbf{X}_{n}=\mathbf{A}_{L}^{n} \mathbf{X}_{0}+\mu\left(\mathbf{I}-\mathbf{A}_{L}^{n}\right) \mathbf{B}_{L} \mathbf{C}
$$


Now consider the following equation where $\mathbf{X}_{0}^{*}=[-\varepsilon, r]^{T}$ and $r$ is an arbitrary real number.

$$
\begin{array}{ll} 
& \mathbf{A}^{n} \mathbf{X}_{0}+\mu\left(\mathbf{B}_{L}-\mathbf{A}_{L}^{n} \mathbf{B}_{L}\right) \mathbf{C}=\mathbf{X}_{0}^{*} \\
\Longrightarrow & \mathbf{A}_{L}^{n}\left(\mathbf{X}_{0}-\mu \mathbf{B}_{L} \mathbf{C}\right)=\mathbf{X}_{0}^{*}-\mu \mathbf{B}_{L} \mathbf{C} \\
\Longrightarrow & \mathbf{A}_{L}^{n} \mathbf{Y}_{0}=\mathbf{Y}_{0}^{*}
\end{array}
$$

where $\mathbf{Y}_{0}=\mathbf{X}_{0}-\mu \mathbf{B}_{L} \mathbf{C}$ and $\mathbf{Y}_{0}^{*}=\mathbf{X}^{*}-\mu \mathbf{B}_{L} \mathbf{C}$.

The above equation (6) implies

$$
\left(\mathbf{P}_{L}^{-1} \mathbf{D}_{L}^{n} \mathbf{P}_{L}\right) Y_{0}=\mathbf{Y}_{0}^{*}
$$

Now let $\mathbf{P}_{L}=\left[\begin{array}{cc}p_{l 1} & p_{l 2} \\ p_{l 3} & p_{l 4}\end{array}\right], \mathbf{Y}_{0}=\left[y_{01}, y_{02}\right]^{T}, \mathbf{Y}_{0}^{*}=\left[y_{01}^{*}, y_{02}^{*}\right]^{T}$. Here we already know all the values of $p_{l 1}, p_{l 2}, p_{l 3}, p_{l 4}, y_{01}, y_{02}, y_{01}^{*}$. Only $y_{02}^{*}$ is unknown as it is a function of $r$, where $r$ is an arbitrary real number.

Then we can find the minimum value of $n$ by considering the following inequality

$$
\mathbf{D}_{L}^{n}\left(\mathbf{P}_{L} \mathbf{Y}_{0}\right) \leq \mathbf{P}_{L} \mathbf{Y}_{0}^{*}
$$

Here we consider that if $\mathbf{X}=\left[x_{1}, x_{2}\right]^{T}$ and $\mathbf{Y}=\left[y_{1}, y_{2}\right]^{T}$ then $\mathbf{X} \leq \mathbf{Y}$ implies $x_{1} \leq y_{1}$. Then from the equation (7) we arrive at the following system of equations

$$
\begin{aligned}
& f_{l 1} \lambda_{1 L}^{n}+f_{l 2} \lambda_{2 L}^{n}=y_{01}^{*} \\
& f_{l 3} \lambda_{1 L}^{n}+f_{l 4} \lambda_{2 L}^{n}=y_{02}^{*}
\end{aligned}
$$

where each $f_{l i}, \quad i=1,2,3,4$, is a function of $p_{l 1}, p_{l 2}, p_{l 3}, p_{l 4}, y_{01}, y_{02}$ and since all the values of these variables are already known therefore we obtain each $f_{l i}, \quad i=1,2,3,4$, as a constant in (9) and (10). Therefore we rewrite the above system of equations as

$$
\left[\begin{array}{ll}
f_{l 1} & f_{l 2} \\
f_{l 3} & f_{l 4}
\end{array}\right]\left[\begin{array}{l}
\lambda_{1 L}^{n} \\
\lambda_{2 L}^{n}
\end{array}\right]=\left[\begin{array}{l}
y_{01}^{*} \\
y_{02}^{*}
\end{array}\right]
$$

This implies

$$
\left[\begin{array}{l}
\lambda_{1 L}^{n} \\
\lambda_{2 L}^{n}
\end{array}\right]=\left[\begin{array}{ll}
f_{l 1} & f_{l 2} \\
f_{l 3} & f_{l 4}
\end{array}\right]^{-1}\left[\begin{array}{l}
y_{01}^{*} \\
y_{02}^{*}
\end{array}\right]
$$

Therefore we have the following equation

$$
\lambda_{1 L}^{n}=k_{l 1} y_{01}^{*}+k_{l 2} y_{02}^{*}
$$


where

$$
\left[\begin{array}{ll}
f_{l 1} & f_{l 2} \\
f_{l 3} & f_{l 4}
\end{array}\right]^{-1}=\left[\begin{array}{ll}
k_{l 1} & k_{l 2} \\
k_{l 3} & k_{l 4}
\end{array}\right] .
$$

If $n=n_{1}$ is a solution of this equation (11) then

$$
n_{1} \leq \frac{\ln \left(k_{l 1} y_{01}^{*}+k_{l 2} y_{02}^{*}\right)}{\ln \left(\lambda_{1 L}\right)}
$$

satisfies the inequality (8).

Now if

$$
n_{1}^{*}=\inf _{r \in \mathbb{R}} \frac{\ln \left(k_{l 1} y_{01}^{*}+k_{l 2} y_{02}^{*}\right)}{\ln \left(\lambda_{1 L}\right)},
$$

then the minimum number of iterations needed for an orbit of (3) to escape from the region $R_{0}$ under the repeated application of $M_{L}$ is

$$
n_{R_{0}}=\left\lfloor n_{1}^{*}\right\rfloor+1,
$$

where \lfloor\rfloor denotes the floor function, i.e., $\lfloor x\rfloor$ denotes the greatest integer not greater than $x$.

\section{PROBABILITIES OF CONVERGENCE AND NON-CONVERGENCE OF AN ORBIT}

Next suppose that $\xi_{n}$ follows a probability distribution $h(x)$ inside the interval $(-\varepsilon, \varepsilon)$. Then

$$
\int_{-\varepsilon}^{\varepsilon} h(x) d x=1
$$

Let us start iterating from any point $\mathbf{X}_{0}=\left(x_{0}, y_{0}\right) \in R_{I}$. Suppose at any $k$-th instant we denote the position of the border by $x=x_{b_{k}}$, then there may arise two possibilities:

1. $x_{k}<x_{b_{k}}$, where $x_{k}$ denotes the $x$-coordinate of the $k$-th iterate $\mathbf{X}_{k}=\left(x_{k}, y_{k}\right)$.

2. $x_{k} \geq x_{b_{k}}$, where $x_{k}$ denotes the $x$-coordinate of the $k$-th iterate $\mathbf{X}_{k}=\left(x_{k}, y_{k}\right)$.

Next we consider the probabilities of the above two situations at any instant. The probability of the first possibility at any $k$-th instant is

$$
P\left(x_{k}<x_{b_{k}}\right)=\int_{x_{k}}^{\varepsilon} h(x) d x
$$


Whereas the probability of the second situation at any $k$-th instant of time is

$$
P\left(x_{k} \geq x_{b_{k}}\right)=\int_{-\varepsilon}^{x_{k}} h(x) d x
$$

Now we first compute the probability that an orbit of (3) starting from $\mathbf{X}_{0} \in R_{I}$ escapes from the region $R_{0}$ under only the repeated application of the map $M_{L}$. In that case at every iteration we should have $x_{i}<x_{b_{i}}$ for all $i=1,2, \cdots, n_{R_{0}}$, where $x=x_{b_{i}}$ is the position of the switching surface and $x_{i}$ denotes the $x$-coordinate of the corresponding iterate $X_{i}=\left(x_{i}, y_{i}\right)$. In such a case, at any $i$-th iteration the probability of $x_{i}<x_{b_{i}}$ is

$$
P\left(x_{i}<x_{b_{i}}\right)=\int_{P_{x}}^{\varepsilon} h(x) d x
$$

where

$$
\mathbf{P}=\left[\mu\left(\frac{1-\mathbf{A}_{L}^{i}}{1-\mathbf{A}_{L}}\right) \mathbf{C}+\mathbf{A}_{L}^{i} \mathbf{X}_{0}\right], \quad \mathbf{Q}=\left[\mu\left(\frac{1-\mathbf{A}_{R}^{i}}{1-\mathbf{A}_{R}}\right) \mathbf{C}+\mathbf{A}_{R}^{i} \mathbf{X}_{0}\right]
$$

and $P_{x}$ and $Q_{x}$ denote the $x$-component of the column vectors $\mathbf{P}$ and $\mathbf{Q}$ respectively.

As the $\xi_{n}$ 's in (3) are independent, the probability that an orbit of (3) starting from $X_{0} \in R_{I}$ escapes from the region $R_{0}$ in $n_{R_{0}}$ number of iterations is

$$
\begin{aligned}
P\left(\bigcap_{i=0}^{n_{R_{0}}}\left(x_{i}<x_{b_{i}}\right)\right. & =\prod_{i=0}^{n_{R_{0}}} P\left(x_{i}<x_{b_{i}}\right) \\
& =\prod_{i=0}^{n_{R_{0}}} \int_{P_{x}}^{\varepsilon} h(x) d x
\end{aligned}
$$

Therefore (17) gives the probability that an orbit of (3) starting from $X_{0} \in R_{I}$, leaves $R_{0}$ in $n_{R_{0}}$ steps.

Now if the orbit starts from an initial point $X_{0} \in R_{I}$ then let

- $P_{\mathbf{X}_{0}}$ denote the probability that the orbit converges on the stable fixed point $L^{*}$;

- $P_{\mathbf{X}_{0}}^{*}$ denote the probability that the orbit does not converge on the stable fixed point $L^{*}$.

Therefore we have the inequality

$$
P_{\mathbf{X}_{0}}>\prod_{i=0}^{n_{R_{0}}} \int_{P_{x}}^{\varepsilon} h(x) d x
$$


Now we know that repeated application of $M_{R} n_{\mathbf{X}_{0}}$ times on some $\mathbf{X}_{0} \in R_{I}$ will throw the $n_{\mathbf{X}_{0}}$-th iterate $\mathbf{X}_{n_{x_{0}}}$ out of the basin of attraction of $L^{*}$ as well as out of the region of variation of the border forever. Therefore if $P_{\mathbf{X}_{0}}^{*}$ denotes the probability of convergence to some other attractor except $\mathbf{L}^{*}$ then we have,

$$
P_{\mathbf{X}_{0}}^{*}>\prod_{i=0}^{n \mathbf{x}_{0}} \int_{-\varepsilon}^{Q_{x}} h(x) d x
$$

Now as $P_{\mathbf{X}_{0}}+P_{\mathbf{X}_{0}}^{*}=1$, we arrive at the following theorem.

Theorem 4.1. Suppose an orbit of the map (3) starts from any arbitrary point $\mathbf{X}_{0} \in R_{I}$. Let $P_{\mathbf{X}_{0}}$ denote the probability of convergence of the orbit to the stable fixed point $\mathbf{L}^{*}$ and $P_{\mathbf{X}_{0}}^{*}$ denote the probability that the orbit converges to some attractor other than $\mathbf{L}^{*}$. Then $P_{\mathbf{X}_{0}}$ and $P_{X_{0}}^{*}$ satisfy the following two inequalities.

$$
\prod_{i=0}^{n_{R_{0}}} \int_{P_{x}}^{\varepsilon} h(x) d x<P_{\mathbf{x}_{0}}<1-\prod_{i=0}^{n_{\mathbf{x}_{0}}} \int_{-\varepsilon}^{Q_{x}} h(x) d x
$$

and

$$
\prod_{i=0}^{n_{\mathbf{x}_{0}}} \int_{-\varepsilon}^{Q_{x}} h(x) d x<P_{\mathbf{X}_{0}}^{*}<1-\prod_{i=0}^{n_{R_{0}}} \int_{P_{x}}^{\varepsilon} h(x) d x
$$

Again from equation (19) we have the following inequality for all $X_{0} \in R_{I}$.

$$
P_{\mathbf{x}_{0}}^{*}>\prod_{i=0}^{n_{\mathbf{x}_{0}}} \inf _{\mathbf{x}_{0} \in R_{I}}\left[\int_{-\varepsilon}^{Q_{x}} h(x) d x\right]
$$

Now, as

$$
\int_{-\varepsilon}^{Q_{x}} h(x) d x>0
$$

for all $\mathbf{X}_{0} \in R_{I}$, therefore (20) indicates that

$$
P_{\mathbf{X}_{0}}^{*}>0
$$

for all $\mathbf{X}_{0} \in R_{I}$. Hence we also have

$$
P_{\mathbf{X}_{0}}>0
$$

for all $\mathbf{X}_{0} \in R_{0}$.

Therefore the equations (21) and (22) imply that the region $R_{I}$ acts as a non-deterministic basin of attraction since, for an orbit starting inside this region, there are non-zero probabilities of convergence to $\mathbf{L}^{*}$ and also of convergence to some attractor other than $\mathbf{L}^{*}$, including the attractor at infinity. Theorem 4.1 gives an upper and a lower bound of the corresponding probabilities. 


\section{CONCLUSION}

This paper reveals the effect of stochastically moving switching manifold in a class of two dimensional piecewise smooth map. In presence of such kind of varying switching surface there may exist a probabilistic basin of attraction. Any orbit starting inside this probabilistic basin of attraction has non-zero probabilities of convergence (to a stable fixed point) as well as of divergence (to infinity). Since the dynamics of all physical systems are contaminated by noise, the appearance of this kind of non-deterministic basin of attraction is a definite possibility. The existence of this type of non-deterministic basin of attraction in a system may lead to dangerous consequences as the probabilistic basin contains a part of the basin of attraction of the stable fixed point of the original system. If the fluctuation of the border remains unnoticed, a piecewise smooth system may experience collapse even when the initial condition is placed within what is perceived as the basin of attraction of the stable fixed point.

Now as we know that higher dimensional continuous-time nonsmooth systems give rise to higher dimensional piecewise smooth maps (when dynamics of continuous time systems are observed on a Poincare section), therefore the phenomena discussed above has significant importance in case of continuous time nonsmooth engineering systems also. Lack of stability is likely to occur in such systems also, if any switching surface vibrates stochastically inside a small region of the phase space. As most of the nonsmooth engineering systems are continuous-time systems, therefore a future research scope will be to investigate the stability of continuous-time nonsmooth systems in presence of randomly varying switching surfaces.

\section{REFERENCES}

[1] S. Banerjee and C. Grebogi. Border collision bifurcations in two-dimensional piecewise smooth maps. Physical Review E, 59(4):4052, 1999.

[2] S. Banerjee and G. C. Verghese. Nonlinear phenomena in power electronics. IEEE, 1999.

[3] S. Banerjee, J. A. Yorke, and C. Grebogi. Robust chaos. Physical Review Letters, 80(14):3049, 1998.

[4] M. Bernardo, C. Budd, A. R. Champneys, and P. Kowalczyk. Piecewise-smooth dynamical systems: theory and applications, volume 163. Springer Science \& Business Media, 2008. 
[5] P. Casini and F. Vestroni. Characterization of bifurcating non-linear normal modes in piecewise linear mechanical systems. International Journal of Non-Linear Mechanics, 46(1):142-150, 2011.

[6] M. Di Bernardo, P. Kowalczyk, and A. Nordmark. Bifurcations of dynamical systems with sliding: derivation of normal-form mappings. Physica D: Nonlinear Phenomena, 170(3-4):175-205, 2002.

[7] P. Glendinning. The border collision normal form with stochastic switching surface. SIAM Journal on Applied Dynamical Systems, 13(1):181-193, 2014.

[8] D. Mandal and S. Banerjee. Effect of stochastically moving border on basins of attraction in a class of piecewise smooth maps. Physics Letters A, 381(27):2161-2166, 2017.

[9] H. E. Nusse and J. A. Yorke. Border-collision bifurcations including "period two to period three" for piecewise smooth systems. Physica D: Nonlinear Phenomena, 57(1-2):39-57, 1992.

[10] M. A. Savi, S. Divenyi, L. F. P. Franca, and H. I. Weber. Numerical and experimental investigations of the nonlinear dynamics and chaos in non-smooth systems. Journal of Sound and Vibration, 301(1-2):59-73, 2007. 